\title{
A Novel Modulation Classification Approach Using Gabor Filter Network
}

\author{
Sajjad Ahmed Ghauri, ${ }^{1,2,3}$ Ijaz Mansoor Qureshi, \\ Tanveer Ahmed Cheema, ${ }^{1,5}$ and Aqdas Naveed Malik ${ }^{3,5}$ \\ ${ }^{1}$ ISRA University, Islamabad 44000, Pakistan \\ ${ }^{2}$ School of Engineering \& Applied Sciences (SEAS), ISRA University, Islamabad Campus, I/10 Markaz, Islamabad 44000, Pakistan \\ ${ }^{3}$ International Islamic University, Islamabad 44000, Pakistan \\ ${ }^{4}$ AIR University, Islamabad 44000, Pakistan \\ ${ }^{5}$ Institute of Signals, Systems and Soft Computing (ISSS), Islamabad, Pakistan
}

Correspondence should be addressed to Sajjad Ahmed Ghauri; sajjad.ghauri.101@gmail.com

Received 24 February 2014; Revised 16 June 2014; Accepted 17 June 2014; Published 14 July 2014

Academic Editor: Nirupam Chakraborti

Copyright (c) 2014 Sajjad Ahmed Ghauri et al. This is an open access article distributed under the Creative Commons Attribution License, which permits unrestricted use, distribution, and reproduction in any medium, provided the original work is properly cited.

\begin{abstract}
A Gabor filter network based approach is used for feature extraction and classification of digital modulated signals by adaptively tuning the parameters of Gabor filter network. Modulation classification of digitally modulated signals is done under the influence of additive white Gaussian noise (AWGN). The modulations considered for the classification purpose are PSK 2 to 64, FSK 2 to 64, and QAM 4 to 64 . The Gabor filter network uses the network structure of two layers; the first layer which is input layer constitutes the adaptive feature extraction part and the second layer constitutes the signal classification part. The Gabor atom parameters are tuned using Delta rule and updating of weights of Gabor filter using least mean square (LMS) algorithm. The simulation results show that proposed novel modulation classification algorithm has high classification accuracy at low signal to noise ratio (SNR) on AWGN channel.
\end{abstract}

\section{Introduction}

Digital modulation is an important factor in communication system. Identification of received signal modulation in the presence of channel noise in noncooperative communication is a complex issue. Before demodulation of received signal, modulation classification is done. Modulation classification is a technique that allows receiver to become cognizant of current status of transmitted data and channel. Applications of modulation classification (MC) are in commercial sector (interference identification, spectrum management), military domain, and software defined radio (SDR). If receivers classify the modulation scheme successfully, the SDR can be used as a receiver for modification of demodulation part. The $\mathrm{MC}$ has applications also in cognitive radios (CR).

After the detection of received signal and before the demodulation of received signal, modulation classification process is done. Modulation classification process involves two steps; the first is feature extraction of the transmitted signal and the second is classification of the signal based upon feature extraction. In the literature, various methods have been proposed for classification/identification of different modulation formats [1]. Basically, automatic modulation classification process is distributed in two approaches [2]: (1) $\log$ likelihood function based decision theoretic approach; (2) extraction of features based pattern recognition approach.

Log likelihood function based decision theoretic approach has been proposed in [3-5]. This approach is basically a function of symbols which are to be transmitted, as well as channel parameter. The log likelihood function is calculated under each modulation format (hypothesis). The modulation format which maximizes the likelihood function is the decision. The log likelihood function based decision algorithms are optimal because they minimize the probability of error in classification of signal, but they are computationally complex. The log likelihood function based 
decision theoretic approach requires a priori knowledge about the signal. The likelihood functions become tussle and hard to implement because of channel conditions. Extraction of features based pattern recognition approach is also known as feature based (FB) approach. The decision is made on the observation of the extracted feature set. FB approach is suboptimal method with reasonable computational complexity as compared to log likelihood based algorithms and also easy to implement $[6,7]$.

In the literature, the feature which was extracted from the transmitted signal is of many types such as higher order moments (HOM), higher order cummulants (HOC) up to 8th order (HOM) [1], spectral features $\left(\sigma_{\text {ap }}, \sigma_{\text {dp }}, \sigma_{\text {aa }}, \sigma_{\text {af }}\right.$, $\sigma_{\text {fn }}$, and $\gamma_{\max }$ ) [8], and cyclic features (spectral coherence function, cyclic domain profile) [9]. In [1,10], authors present the automatic modulation classification algorithm which uses HOC for estimation of channel and pattern recognition; no a priori information is required. In [11], HOC up to 4th order are used for classification of modulations over an AWGN channel. The features utilized in $[9,12,13]$ are spectral features $\left(\sigma_{\mathrm{ap}}, \sigma_{\mathrm{dp}}, \sigma_{\mathrm{aa}}, \sigma_{\mathrm{af}}, \sigma_{\mathrm{fn}}\right.$, and $\left.\gamma_{\max }\right)$ and cyclic features (spectral coherence function, cyclic domain profile). A summary of proposed algorithms based on timefrequency analysis, wavelet transform, higher order statistics (cummulants and moments), cyclo-stationarity properties and on spectral properties for modulation classification are in [14].

The modulation identification of BPSK, QPSK, 16-PSK, 216QAM, GMSK, and MSK modulations schemes under the effect of noisy channel is considered in [15], using wavelet transform approach and statistical moments as features. The proposed algorithm performs $100 \%$ identification at SNR of $10 \mathrm{~dB}$. A hierarchical cyclostationary based algorithm is proposed to identify the wide range of unknown modulated signals presented in [16]. The authors also assume no a priori information such as carrier frequency and carrier phase. The modulation formats to be classified are AM, BPSK, OFDM, CDMA, 4-8 ASK, 2-16 PSK, 16, and 64 QAM modulation formats. The performance of proposed algorithm is investigated on fading channels. The proposed algorithm in [17] uses higher order moments of continuous wavelet transform (CWT) as a feature set. The classifier used is multilayer feed forward neural network using resilient back propagation algorithm. The modulation formats considered are M-ary shift keying without any priori information. The performance of algorithm is evaluated on AWGN channel as well as fading channels. In [18], FSK and AM signals are jointly detected and classified using first order cyclostationarity. The algorithm only requires approximate information about signal band width and carrier frequency and there is no need for time recovery in the proposed algorithm. In [19], features are extracted based upon autocorrelation and cyclic autocorrelation for cyclic prefix guard time interval OFDM signals to estimate the useful time interval, the cyclic prefix duration, and the number of subcarriers in frequency selective channels. Authors use time frequency analysis to extract features in [20]. These extracted features are used for classification purpose. The proposed algorithm has three main tools; a TF tool which computes the TF transform, extraction of features which gives main characteristics of signal, and classifier part which discriminates with the help of features. The modulation classification for the wireless sensor networks is carried out in [21] using multisensor fusion based method. The classification performance is investigated on AWGN channel and fading channel. The a priori information about the signal such as timing synchronization, phase jitter, phase offset, and frequency offset is considered for evaluation of correct classification. The first signal classification is done using $2 \mathrm{nd}$, 4 th, and 6th order cummulants and then kernel thought is used to map the feature to higher dimensional space and optimum hyper plane is constructed using SVM to classify the signals in [22]. The classifier based on back propagation neural network (BPNN) and trained by improved particle swarm optimization (PSO) which is used to optimal weights and threshold for BPNN is proposed in [23]. The recognized modulation formats are 2ASK, 4ASK, 2FSK, 4FSK, BPSK, and QPSK. The classifier designed for space time block codes (STBC) system using multidimensional independent component analysis (ICA) is proposed in [24]. The classifier is also based on maximum likelihood on the condition of virtual channel matrix. The features extracted for modulation identification are received signal power distribution in [25]. The classifier identifies only six modulation formats. Higher order cummulants up to 4 th and 6th order are used to classify the modulation formats such as BPSK, QPSK, and higher order QAM's [26]. Cyclostationary features are also used to classify the BPSK and non-BPSK, at last maximum likelihood detection algorithm is performed to further classification of QPSK and 16-QAM. The modulation classification is done without having any information about noise power, energy of the transmitted signal, and channel coefficients [27]. The modulation classification is carried out by minimizing the distance of log likelihood and expected log likelihood of the received data. Modulation classification is considered on frequency selective fading channel using Gibbs sampling method based on latent Dirichlet Bayesian network in [28]. Blind modulation classification problem is considered under the effect on frequency selective fading channels in [29]. The features used for classification are correlation function of the received signal. The modulation recognition focuses on pattern recognition method proposed in [30]; the main purpose is to demonstrate the possibility of recognizing digital modulation formats at lower SNRs. The method of modulation classification involves computation of the empirical characteristics function (ECF) from the received signal samples and employing constrained least squares (CLS) filtering in frequency domain [31].

For signal classification and representations, adaptive time frequency analysis including wavelet based filter bank [32] and Gabor based filter banks were used in [33, 34]. Recently, in [35], authors proposed a Gabor atom based upon neural network (GNN) for feature extraction and signal classification. Efficient feature extraction and higher recognition rate have been achieved using GNN. However, GNN consists of two layers; the first layer is feature extraction and the second layer is of signal classification. Using degree of non-stationarity, the Gabor filter is proposed in [36], for efficient classification. 
In this paper, the authors have proposed joint approach for feature extraction and classification for multisignal vector. Gabor filter based approach is used to classify the digital modulated signal in the presence of AWGN channel. The Gabor filter parameters are adjusted adaptively using the Delta rule. The weights of the adaptive filter are adjusted using least mean square (LMS) algorithm. The digital modulations considered in this paper are PSK2, PSK4, PSK8, PSK16, PSK32, PSK64, FSK2, FSK4, FSK8, FSK16, FSK32, FSK64, QAM2, QAM4, QAM8, QAM 16, QAM 32, and QAM 64. The proposed algorithm gives high classification accuracy at lower SNRs. The mean square error (MSE) for training of Gabor filter network versus number of iterations as well as versus SNR for considered modulations is also shown. The simulation results for testing of proposed algorithm show high classification accuracy.

The rest of the paper is organized as follows. Section 2 represents the system model. Section 3 represents the Gabor filter for classification and feature extraction. In Section 4, Gabor filter training and testing algorithm is presented. Section 5 discusses the performance of proposed classifier in the presence of AWGN, while the whole paper is concluded in Section 5.

\section{System Model}

The generalized expression for signal received is given by

$$
r(n)=x(n)+g(n),
$$

where $r(n)$ is complex baseband envelop of received signal, $g(n)$ is the additive white Gaussian noise with zero mean and a variance of $\sigma_{g}{ }^{2}$, and $x(n)$ is given by

$$
x(n)=\alpha e^{i\left(w_{o} n T+\theta_{n}\right)} \sum_{j=-\infty}^{j=\infty} x(l) h\left(n \tau-j \tau+\epsilon_{T} \tau\right),
$$

where $x(l)=$ input symbol sequence which is drawn from set of $M$ constellations of known symbols and it is not necessary that symbols are equiprobable, $\alpha=$ amplitude of signal, $w_{o}=$ angular frequency offset constant, $\tau=$ symbol spacing, $\theta_{n}=$ the phase jitter which varies from symbol to symbol, $h(\cdots)=$ channel effects, and $\epsilon_{T}=$ the timing jitter.

The system model for classification of modulation signals is shown in Figure 1. First feature extracted from the received signal which is corrupted by additive white Gaussian noise, after extraction of these features the classification is based upon the feature extracted. The received signal may be PSK, FSK, or QAM modulated.

\section{Gabor Filter for Classification and Feature Extraction}

Gabor atom is efficient tool for feature extraction. The Gabor atom in simple form can be written as

$$
g_{c, \sigma, f}(t)=\frac{1}{\sqrt{\sigma}} g\left(\frac{t-c}{\sigma}\right) e^{j f t}
$$

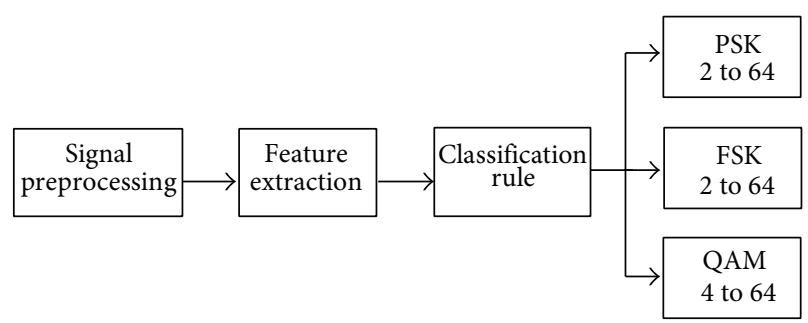

FIGURE 1: System model for modulation classification.

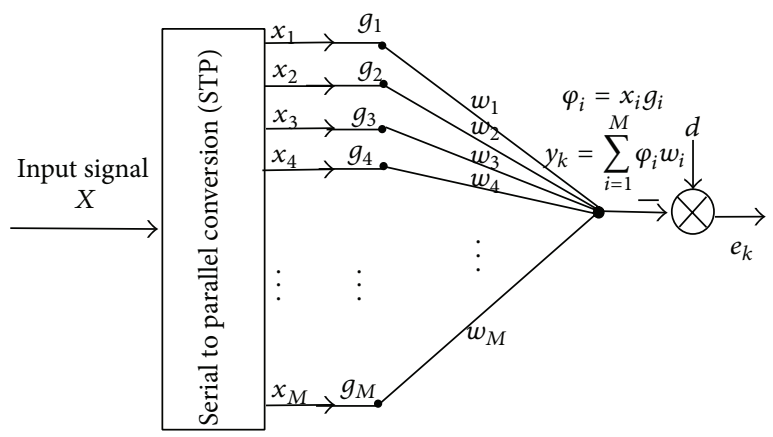

Figure 2: Gabor filter network with input layer which is feature extraction part; weights and output layer are linear classification part.

where $g(t)=2^{1 / 4} e^{-\pi t^{2}}$ and $c, \sigma$, and $f$ are shift parameter, scale parameter, and modulation parameter, respectively.

In Figure 2, Gabor filter network is shown which has twolayer filter. The input to Gabor filter network is first serial to parallel converted $\left\{x_{i}, i=1,2,3, \ldots, M\right\}$ and outputs are $\left\{y_{k}\right.$, $k=1,2,3, \ldots, N\}$. Let $\left\{g_{i}, i=1,2,3, \ldots, M\right\}$ be the $i$ th class Gabor atom and be defined as

$$
g_{i}(t)=\frac{1}{\sqrt{\sigma_{i}}} g\left(\frac{t-c_{i}}{\sigma_{i}}\right) e^{j f_{i} t} .
$$

The Gabor atom parameters $(c, \sigma$, and $f$ ) are required to be adjusted until some cost function is minimized.

The input layer has $M$ nodes $\varphi_{1}, \varphi_{2}, \varphi_{3}, \ldots, \varphi_{M}$ also called Gabor nodes. The output of the $i$ th Gabor atom node is $\varphi_{i}$ corresponding to input signal $x_{i}$. Thus, output of Gabor atom is defined as

$$
\begin{gathered}
\varphi_{i}=\left|\left\langle g_{i}, x_{i}\right\rangle\right| \\
\varphi_{i}=\left|\int \frac{1}{\sqrt{\sigma_{i}}} g^{*}\left(\frac{t-c_{i}}{\sigma_{i}}\right) e^{-j f_{i} t} x_{i}(t) d t\right| .
\end{gathered}
$$

The output layer consists of $N$ nodes $\left\{y_{k}, k=1,2,3, \ldots, M\right\}$ and for convenience $N$ is usually set to 1 . The output of the Gabor atom node $\varphi_{i}$ in the input layer is weighted by $w_{i}$; that is,

$$
y_{k n}=\sum_{i=1}^{M} \varphi_{i n} w_{i n}, \quad n=1,2,3, \ldots, N .
$$

Gabor filter network consists of two layers: the input layer is feature extraction and the second layer has Gabor filter weights which constitutes the linear classification part. 


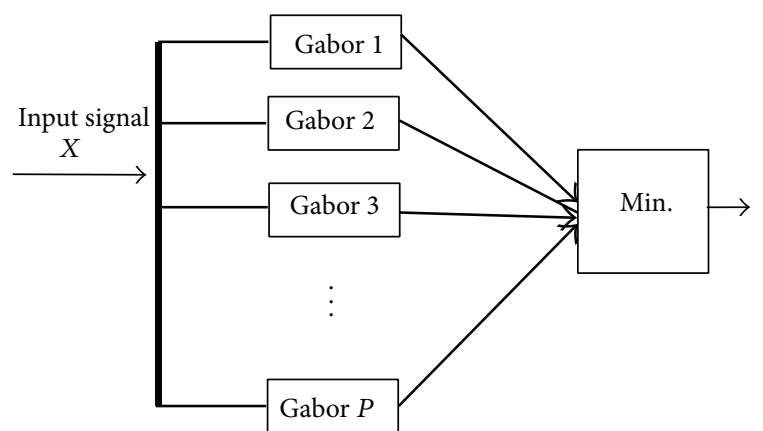

FIgURE 3: Testing scheme for modulation classification.

Feature extraction using Gabor filter network is that both Gabor atom parameters and Gabor filter weights are adjusted to minimize the sum of squared error. The difference between the desired outputs $d_{k}$ and actual output of Gabor filter $y_{k}$ is defined as

$$
e_{k}=d_{k}-y_{k}
$$

In [35], the Gabor atom parameters and neural network weights are adjusted simultaneously in training phase. Such a joint updating of Gabor atom parameters and neural networks weights show some deficiencies. These deficiencies will cope when Gabor atom parameters and neural networks weights are adjusted separately [36].

In training phase of modulation classification, the two adaptive algorithms are performed by Gabor filter network: (1) the updating of Gabor atom parameters $(c, \sigma$, and $f) ;(2)$ for given set of Gabor atom parameters, algorithm updates the weight of Gabor filter.

In testing phase, shown in Figure 3, the modulated signal may be PSK 2 to 64, FSK 2 to 64, and QAM 2 to 64. The modulated signal is passed through Gabor filter network that updates 4 parameters $(c, \sigma, f$, and $w)$ and based upon these parameters error is calculated. The minimum error corresponds to decision about the receive signal modulation.

\section{Testing and Training of Proposed Algorithm}

The training of Gabor filter network is partitioned into two phases: training of Gabor atom parameters $(c, \sigma, f$, and $w)$ in the first phase and training the weights of adaptive filter in second phase. The parameters of Gabor atom parameters $(c, \sigma, f, w)$ are tuned according to Delta rule and weights of adaptive filter are adjusted by least means square algorithm.

Let $\gamma_{i}$ denote one of $i$ th Gabor node parameters including shift parameter $c_{i}$, scale parameter $\sigma_{i}$, and modulation parameter $f_{i}$. According to Delta rule,

$$
\Delta \gamma_{i}=-\eta \frac{\partial J(k)}{\partial \gamma_{i}}
$$

where $\eta$ is learning rate.

The cost function is square of difference between desired response and output of Gabor function; that is,

$$
J(k)=(d(k)-y(k))^{2} .
$$

The partial derivatives of cost function with respect to shift parameter $c_{i}$, scale parameter $\sigma_{i}$, and modulation para + meter $f_{i}$ are as follows:

$$
\begin{aligned}
\Delta c_{i} & =c_{i}(n+1)-c_{i}(n) \\
& =-\frac{\eta_{c}}{2} \frac{\partial J(k)}{\partial c_{i}} \\
& =-\frac{\eta_{c}}{2}\left[\frac{\partial J(k)}{\partial \varphi_{i}} \frac{\partial \varphi_{i}}{\partial c_{i}}\right], \\
\Delta \sigma_{i} & =\sigma_{i}(n+1)-\sigma_{i}(n) \\
& =-\frac{\eta_{\sigma}}{2} \frac{\partial J(k)}{\partial \sigma_{i}} \\
& =-\frac{\eta_{\sigma}}{2}\left[\frac{\partial J(k)}{\partial \varphi_{i}} \frac{\partial \varphi_{i}}{\partial \sigma_{i}}\right], \\
\Delta f_{i} & =f_{i}(n+1)-f_{i}(n) \\
& =-\frac{\eta_{f}}{2} \frac{\partial J(k)}{\partial f_{i}} \\
& =-\frac{\eta_{f}}{2}\left[\frac{\partial J(k)}{\partial \varphi_{i}} \frac{\partial \varphi_{i}}{\partial f_{i}}\right] .
\end{aligned}
$$

From (9),

$$
\begin{aligned}
& \frac{\partial J(k)}{\partial \varphi_{i}}=\frac{\partial}{\partial \varphi_{i}}\left[(d(k)-y(k))^{2}\right], \\
& \frac{\partial J(k)}{\partial \varphi_{i}}=2[d(k)-y(k)] \frac{\partial}{\partial \varphi_{i}}(d(k)-y(k)), \\
& \frac{\partial J(k)}{\partial \varphi_{i}}=-2[d(k)-y(k)] \frac{\partial}{\partial \varphi_{i}}(y(k)) .
\end{aligned}
$$

From (6),

$$
\begin{aligned}
& \frac{\partial}{\partial \varphi_{i}}(y(k))=\frac{\partial}{\partial \varphi_{i}}\left[\sum_{j=1}^{M} \varphi_{j} w_{j}\right], \\
& \frac{\partial}{\partial \varphi_{i}}(y(k))=\sum_{j=1}^{M} \delta_{i j} w_{j}, \\
& \frac{\partial}{\partial \varphi_{i}}(y(k))=w_{i} .
\end{aligned}
$$

Putting (17) into (16), we get

$$
\frac{\partial J(k)}{\partial \varphi_{i}}=-2(d(k)-y(k)) w_{i}
$$


From (11), (13), and (15),

$$
\begin{aligned}
\Delta c_{i} & =\eta_{c}(d(k)-y(k)) w_{i} \frac{\partial \varphi_{i}}{\partial c_{i}}, \\
\Delta \sigma_{i} & =\left[\eta_{\sigma}(d(k)-y(k)) w_{i}\right] \frac{\partial \varphi_{i}}{\partial \sigma_{i}}, \\
\Delta f_{i} & =\left[\eta_{f}(d(k)-y(k)) w_{i}\right] \frac{\partial \varphi_{i}}{\partial f_{i}}, \\
g_{i} & =\frac{1}{\sqrt{\sigma_{i}}} e^{-\pi\left(\left(t-c_{i}\right) / \sigma_{i}\right)^{2}} \cos \left(f_{i} t\right) .
\end{aligned}
$$

From (5),

$$
\varphi_{i}=\left|x_{i} \frac{1}{\sqrt{\sigma_{i}}} e^{-\pi\left(\left(t-c_{i}\right) / \sigma_{i}\right)^{2}} \cos \left(f_{i} t\right)\right| .
$$

For real valued signals, Gabor atom is also real, in such case.

The partial derivatives of $\varphi_{i}$ with respect to shift parameter $c_{i}$, scale parameter $\sigma_{i}$, and modulation parameter $f_{i}$ are as follows:

$$
\begin{aligned}
& \frac{\partial \varphi_{i}}{\partial c_{i}}=\frac{\partial}{\partial c_{i}}\left(x_{i} g_{i}\right) \\
& =\frac{\partial}{\partial c_{i}}\left[x_{i} \frac{1}{\sqrt{\sigma_{i}}} e^{-\pi\left(\left(t-c_{i}\right) / \sigma_{i}\right)^{2}} \cos \left(f_{i} t\right)\right] \\
& =\frac{x_{i}}{\sqrt{\sigma_{i}}} \cos \left(f_{i} t\right) \\
& \times\left[e^{-\pi\left(\left(t-c_{i}\right) / \sigma_{i}\right)^{2}} \times-\pi\left(2\left(\frac{t-c_{i}}{\sigma_{i}}\right)\right)\left(-\frac{1}{\sigma_{i}}\right)\right] \\
& =\frac{x_{i}}{\sqrt{\sigma_{i}}} \cos \left(f_{i} t\right)\left[e^{-\pi\left(\left(t-c_{i}\right) / \sigma_{i}\right)^{2}}\left(2 \pi\left(\frac{t-c_{i}}{\sigma_{i}^{2}}\right)\right)\right] \\
& =\frac{x_{i}}{\sqrt[5]{\sigma_{i}}} \cos \left(f_{i} t\right) 2 \pi\left(t-c_{i}\right) e^{-\pi\left(\left(t-c_{i}\right) / \sigma_{i}\right)^{2}}, \\
& \frac{\partial \varphi_{i}}{\partial \sigma_{i}}=\frac{\partial}{\partial \sigma_{i}}\left[x_{i} \frac{1}{\sqrt{\sigma_{i}}} e^{-\pi\left(\left(t-c_{i}\right) / \sigma_{i}\right)^{2}} \cos \left(f_{i} t\right)\right] \\
& =x_{i} \cos \left(f_{i} t\right) \\
& \times\left[\frac{1}{\sqrt{\sigma_{i}}} e^{-\pi\left(\left(t-c_{i}\right) / \sigma_{i}\right)^{2}} \times-\pi\left(2\left(\frac{t-c_{i}}{\sigma_{i}}\right)\right)\right. \\
& \left.\times\left(-\frac{\left(t-c_{i}\right)}{\sigma_{i}^{2}}\right)+e^{-\pi\left(\left(t-c_{i}\right) / \sigma_{i}\right)^{2}} \times-\frac{1}{2} \sigma_{i}^{-3 / 2}\right] \\
& =\frac{x_{i} \cos \left(f_{i} t\right)}{\sqrt{\sigma_{i}}} e^{-\pi\left(\left(t-c_{i}\right) / \sigma_{i}\right)^{2}}\left[\frac{2 \pi\left(t-c_{i}\right)^{2}}{\sigma_{i}^{3}}-\frac{1}{2 \sigma_{i}}\right] \\
& \frac{\partial \varphi_{i}}{\partial f_{i}}=\frac{\partial}{\partial f_{i}}\left[x_{i} \frac{1}{\sqrt{\sigma_{i}}} e^{-\pi\left(\left(t-c_{i}\right) / \sigma_{i}\right)^{2}} \cos \left(f_{i} t\right)\right] \\
& =-\frac{t}{\sqrt{\sigma_{i}}} x_{i} e^{-\pi\left(\left(t-c_{i}\right) / \sigma_{i}\right)^{2}} \sin \left(f_{i} t\right) .
\end{aligned}
$$

The Updating of Gabor atom parameters (shift parameter $c_{i}$, scale parameter $\sigma_{i}$, and modulation parameter $f_{i}$ ) according to Delta rule is as follows:

$$
\begin{aligned}
& \Delta c_{i}=\eta_{c}(d(k)-y(k)) w_{i} \\
& \times\left[\frac{x_{i}}{\sqrt[5]{\sigma_{i}}} \cos \left(f_{i} t\right) 2 \pi\left(t-c_{i}\right) e^{-\pi\left(\left(t-c_{i}\right) / \sigma_{i}\right)^{2}}\right], \\
& c_{i}(n+1)=c_{i}(n)+\eta_{c}(d(k)-y(k)) w_{i} \\
& \times\left[\frac{x_{i}}{\sqrt[5]{\sigma_{i}}} \cos \left(f_{i} t\right) 2 \pi\left(t-c_{i}\right) e^{-\pi\left(\left(t-c_{i}\right) / \sigma_{i}\right)^{2}}\right], \\
& \Delta \sigma_{i}=\left[\eta_{\sigma}(d(k)-y(k)) w_{i}\right] \\
& \times\left\{\frac{x_{i} \cos \left(f_{i} t\right)}{\sqrt{\sigma_{i}}} e^{-\pi\left(\left(t-c_{i}\right) / \sigma_{i}\right)^{2}}\right. \\
& \left.\times\left[\frac{2 \pi\left(t-c_{i}\right)^{2}}{\sigma_{i}^{3}}-\frac{1}{2 \sigma_{i}}\right]\right\}, \\
& \sigma_{i}(n+1)=\sigma_{i}(n)+\left[\eta_{\sigma}(d(k)-y(k)) w_{i}\right] \\
& \times\left\{\frac{x_{i} \cos \left(f_{i} t\right)}{\sqrt{\sigma_{i}}} e^{-\pi\left(\left(t-c_{i}\right) / \sigma_{i}\right)^{2}}\right. \\
& \left.\times\left[\frac{2 \pi\left(t-c_{i}\right)^{2}}{\sigma_{i}{ }^{3}}-\frac{1}{2 \sigma_{i}}\right]\right\}, \\
& \Delta f_{i}=\left[\eta_{f}(d(k)-y(k)) w_{i}\right] \\
& \times\left\{-\frac{t}{\sqrt{\sigma_{i}}} x_{i} e^{-\pi\left(\left(t-c_{i}\right) / \sigma_{i}\right)^{2}} \sin \left(f_{i} t\right)\right\}, \\
& f_{i}(n+1)=f_{i}(n)+\left[\eta_{f}(d(k)-y(k)) w_{i}\right] \\
& \times\left\{-\frac{t}{\sqrt{\sigma_{i}}} x_{i} e^{-\pi\left(\left(t-c_{i}\right) / \sigma_{i}\right)^{2}} \sin \left(f_{i} t\right)\right\} .
\end{aligned}
$$

Equations (23), (25), and (27) show the updated shift parameter, scale parameter, and modulation parameter of Gabor filter network.

The weights of adaptive filter are updated as follows:

$$
\begin{aligned}
\Delta w_{i} & =w_{i}(n+1)-w_{i}(n) \\
& =-\frac{\eta_{w}}{2} \frac{\partial J(k)}{\partial w_{i}} \\
& =-\frac{\eta_{w}}{2} \frac{\partial}{\partial w_{i}}\left[(d(k)-y(k))^{2}\right] \\
& =\frac{\eta_{w}}{2} 2(d(k)-y(k)) \frac{\partial}{\partial w_{i}} y(k)
\end{aligned}
$$


Step 1. Initialization of Gabor atom parameters (shift parameter $c_{i}$, scale parameter $\sigma_{i}$ and modulation parameter $f_{i}$ ) and weights of Gabor filter $\left(w_{i}\right)$.

Step 2. Calculate the Gabor atom using (4) and using (20), compute all Gabor atom nodes.

Step 3. The Gabor atoms node $\left(\varphi_{i}\right)$ are now input to the Adaptive filter, and adjust the weights of the adaptive filter using LMS (28)-(32).

Step 4. Evaluate error which is defined in (7). If error is less than chosen threshold, then training of algorithm is stopped and save Gabor atom parameters $\left(c_{i}, \sigma_{i}, f_{i}\right)$ and Gabor filter weights $\left(w_{i}\right)$.

Step 5. If error is not less than threshold, repeat Step 3 by using the error calculated in Step 4.

Step 6. Tune the Gabor atom parameters $\left(c_{i}, \sigma_{i}, f_{i}\right)$ using (8), (23), (25) and (27).

Step 7. Save Gabor atom parameters $\left(c_{i}, \sigma_{i}, f_{i}\right)$ and Gabor filter weights $\left(w_{i}\right)$.

Algorithm 1: (Training of Gabor filter Network for Modulation Classification).

Step 1. Input Gabor atom parameters $\left(c_{i}, \sigma_{i}, f_{i}\right)$ and Gabor filter weights $\left(w_{i}\right)$.

Step 2. Calculate the Gabor atom nodes $\left(\varphi_{i}\right)$

Step 3. Evaluate the output of Gabor filter

$$
y_{k}=\sum_{i=1}^{M} \varphi_{i} w_{i}
$$

Step 4. Decision based upon checking all the outputs

Algorithm 2: (Testing of Gabor filter Network for Modulation Classification).

$$
\begin{aligned}
& =\eta_{w}(d(k)-y(k)) \frac{\partial}{\partial w_{i}} y(k), \\
\frac{\partial y(k)}{\partial w_{i}} & =\frac{\partial}{\partial w_{i}}\left[\sum_{j=1}^{M} \varphi_{j} w_{j}\right]=\varphi_{i} .
\end{aligned}
$$

Substituting (30) in (28), we get

$$
\Delta w_{i}=\eta_{w}(d(k)-y(k)) \varphi_{i} .
$$

From (28),

$$
w_{i}(n+1)=w_{i}(n)+\eta_{w}(d(k)-y(k)) \varphi_{i}
$$

Equation (32) shows the weight updating of the adaptive filter using least mean square algorithm.

The proposed algorithm is for feature extraction and classification of modulation formats (PSK 2 to 64, FSK 2 to 64, and QAM 2 to 64) under the influence of AWGN channel. The proposed algorithm is divided in to two phases; the first phase is for the training of Gabor filter network. In training phase, the parameters of Gabor filter network (shift, scale, and modulation) are updated according to delta rule. These parameters are now input to the adaptive filter where weights of adaptive filter are adjusted using least mean square algorithm. The error is now calculated; if error is less than the threshold, training process stops; otherwise, update the Gabor filter parameters and weights of the adaptive filter according to Delta rule and LMS algorithm until the error function is minimized. The second phase is the test phase of the algorithm, where input modulated signal is fed to the trained Gabor filter network. The parameters of Gabor filter network and weights of the adaptive filter are updated and error is calculated. The minimum error corresponds to the desired modulation format.

The proposed algorithm for training and testing of Gabor filter network for the problem of modulation classification is presented as shown in Algorithms 1 and 2.

\section{Simulation Results}

The modulation classification using Gabor filter is evaluated in this section. Firstly, the training of algorithm is presented and then the testing of algorithm in the presence of AWGN channel. The probability of correct classification (PCC) in the presence of AWGN channel is simulated here using Gabor filter network. The modulation schemes considered here are divided in three scenarios, that is, $\{$ PSK2, PSK4, PSK8, PSK16, PSK 32, and PSK64\}, \{FSK2, FSK4, FSK8, FSK16, FSK 32, and FSK64\}, and \{QAM2, QAM4, QAM8, QAM16, QAM32, and QAM64\}. The PCC curves are simulated against number of iterations and SNR for three different modulation scenarios.

Tables 1-3 and Figures 4-9 show the training of Gabor filter network for the considered modulation formats (PSK, FSK, and QAM) up to order 2 to 64. The Gabor filter network parameters (shift, scale, and modulation) are updated according to each of which considered modulation formats using delta rule and also weights are updated for each considered modulation format case using least mean square 
TABLE 1: Updated Gabor filter atom parameters and weights for PSK modulation 2-64.

\begin{tabular}{|c|c|c|c|c|c|}
\hline \multicolumn{6}{|c|}{ Shift parameter $(c)$} \\
\hline PSK2 & PSK4 & PSK8 & PSK16 & PSK32 & PSK64 \\
\hline 5.54 & 5.07 & 5.77 & 5.65 & 4.81 & 5.27 \\
\hline 5.77 & 5.90 & 4.25 & 4.45 & 4.18 & 4.29 \\
\hline 4.69 & 4.24 & 4.33 & 5.20 & 4.56 & 5.56 \\
\hline 4.63 & 4.72 & 4.83 & 4.17 & 4.67 & 4.56 \\
\hline 5.02 & 4.29 & 5.00 & 4.08 & 5.13 & 4.59 \\
\hline 5.03 & 5.18 & 5.68 & 5.89 & 5.23 & 4.79 \\
\hline 5.16 & 5.09 & 5.89 & 4.91 & 5.79 & 4.63 \\
\hline 4.61 & 4.67 & 5.52 & 5.91 & 4.35 & 4.47 \\
\hline 4.48 & 4.59 & 5.13 & 5.62 & 5.61 & 4.47 \\
\hline 5.43 & 5.14 & 5.13 & 4.94 & 4.15 & 4.75 \\
\hline \multicolumn{6}{|c|}{ Scale parameter $(\sigma)$} \\
\hline PSK2 & PSK4 & PSK8 & PSK16 & PSK32 & PSK64 \\
\hline 15.63 & 16.25 & 10.25 & 16.76 & 1.67 & 2.45 \\
\hline 16.03 & 8.57 & 8.17 & 5.18 & 13.53 & 16.21 \\
\hline 14.87 & 14.08 & 2.55 & 18.87 & 6.78 & 5.33 \\
\hline 1.92 & 18.27 & 12.31 & 6.63 & 13.33 & 4.66 \\
\hline 6.44 & 3.16 & 11.89 & 18.48 & 9.38 & 5.94 \\
\hline 13.32 & 3.17 & 4.80 & 6.85 & 11.90 & 2.50 \\
\hline 11.49 & 12.94 & 12.83 & 15.33 & 7.44 & 15.29 \\
\hline 14.39 & 3.33 & 7.33 & 7.58 & 6.03 & 5.21 \\
\hline 19.04 & 14.23 & 7.39 & 5.13 & 18.00 & 12.81 \\
\hline 8.67 & 2.94 & 8.48 & 1.05 & 12.56 & 2.59 \\
\hline \multicolumn{6}{|c|}{ Modulation parameter $(f)$} \\
\hline PSK2 & PSK4 & PSK8 & PSK16 & PSK32 & PSK64 \\
\hline 2.35 & -0.05 & 0.80 & 3.14 & -0.53 & 1.82 \\
\hline-2.72 & -0.56 & -0.37 & -0.53 & 2.90 & -3.02 \\
\hline 1.91 & 2.52 & -1.90 & 0.95 & -1.33 & -2.24 \\
\hline-1.02 & 0.10 & -1.55 & 1.46 & 2.47 & 0.38 \\
\hline 1.73 & 1.08 & 1.83 & 1.51 & -0.75 & -1.89 \\
\hline-2.75 & -1.83 & -2.57 & -1.42 & 2.66 & 1.10 \\
\hline 0.47 & 0.20 & -2.79 & -2.21 & 0.54 & -1.57 \\
\hline 0.32 & 0.11 & -0.93 & -1.56 & -0.94 & -0.55 \\
\hline 1.88 & 0.33 & -2.59 & 0.31 & -1.28 & 0.68 \\
\hline-0.76 & -2.27 & 0.07 & 2.83 & -1.01 & -1.70 \\
\hline \multicolumn{6}{|c|}{ Weights $(w)$} \\
\hline PSK2 & PSK4 & PSK8 & PSK16 & PSK32 & PSK64 \\
\hline 5.059 & -2.625 & -0.747 & -0.119 & 0.837 & -4.310 \\
\hline-1.823 & -1.927 & 0.671 & 0.297 & 1.309 & -3.073 \\
\hline 6.844 & -2.482 & -0.533 & -0.371 & -3.033 & 8.333 \\
\hline 1.847 & 2.528 & 0.499 & -0.356 & -0.894 & -3.802 \\
\hline 5.384 & -0.090 & 0.677 & -0.346 & -1.484 & 1.413 \\
\hline 8.671 & 1.923 & 0.256 & -0.917 & -0.843 & 3.887 \\
\hline 0.058 & -2.857 & -0.703 & -0.455 & 0.449 & -1.762 \\
\hline-1.356 & 1.852 & -0.555 & -0.487 & -1.844 & 2.682 \\
\hline 1.899 & 1.055 & 0.481 & -1.498 & 0.844 & -3.901 \\
\hline-3.603 & -2.714 & 0.402 & -0.664 & 1.832 & 2.549 \\
\hline
\end{tabular}

algorithm. The Gabor atom parameters and weights of Gabor filter $\left(c_{i}, \sigma_{i}, f_{i}\right.$, and $\left.w_{i}\right)$ for the considered modulations are
TABLE 2: Updated Gabor filter atom parameters and weights for FSK modulation 2-64.

\begin{tabular}{|c|c|c|c|c|c|}
\hline \multicolumn{6}{|c|}{ Shift parameter $(c)$} \\
\hline FSK2 & FSK4 & FSK8 & FSK16 & FSK32 & FSK64 \\
\hline 4.25 & 4.36 & 5.35 & 4.67 & 5.74 & 4.22 \\
\hline 4.09 & 4.88 & 5.80 & 4.20 & 4.55 & 5.93 \\
\hline 4.58 & 4.59 & 5.54 & 5.94 & 4.01 & 5.89 \\
\hline 5.50 & 5.81 & 5.71 & 5.35 & 5.57 & 5.25 \\
\hline 4.46 & 5.55 & 4.60 & 5.83 & 4.59 & 5.96 \\
\hline 5.36 & 4.93 & 4.28 & 4.01 & 5.50 & 5.89 \\
\hline 4.27 & 4.49 & 4.16 & 4.31 & 5.15 & 5.95 \\
\hline 4.38 & 5.28 & 4.75 & 5.70 & 5.76 & 5.40 \\
\hline 4.35 & 5.03 & 4.41 & 5.04 & 5.39 & 4.77 \\
\hline 4.38 & 4.29 & 5.58 & 5.97 & 5.31 & 5.84 \\
\hline \multicolumn{6}{|c|}{ Scale parameter $(\sigma)$} \\
\hline FSK2 & FSK4 & FSK8 & FSK16 & FSK32 & FSK64 \\
\hline 4.44 & 6.46 & 6.80 & 17.24 & 19.65 & 12.27 \\
\hline 14.22 & 2.53 & 3.57 & 3.73 & 6.25 & 19.22 \\
\hline 2.11 & 8.02 & 15.44 & 10.10 & 17.67 & 6.86 \\
\hline 5.89 & 10.94 & 4.75 & 11.42 & 4.03 & 6.93 \\
\hline 19.21 & 2.30 & 11.89 & 2.10 & 6.77 & 19.96 \\
\hline 19.44 & 2.78 & 14.26 & 5.46 & 4.12 & 14.04 \\
\hline 17.74 & 4.84 & 11.82 & 10.50 & 3.96 & 5.87 \\
\hline 1.37 & 6.67 & 10.98 & 17.24 & 17.69 & 5.87 \\
\hline 19.53 & 15.99 & 13.96 & 8.73 & 19.91 & 3.74 \\
\hline 12.67 & 3.57 & 1.45 & 14.57 & 3.65 & 19.59 \\
\hline \multicolumn{6}{|c|}{ Modulation parameter $(f)$} \\
\hline FSK2 & FSK4 & FSK8 & FSK16 & FSK32 & FSK64 \\
\hline-0.15 & -2.97 & 2.87 & 1.64 & 2.42 & -1.70 \\
\hline 1.15 & 2.77 & 0.84 & 0.76 & -1.48 & -2.07 \\
\hline 1.22 & 1.82 & 2.69 & -2.54 & -1.23 & -0.84 \\
\hline-1.50 & 2.91 & -0.67 & 2.37 & 0.77 & 3.10 \\
\hline 2.87 & 1.94 & -0.99 & -2.95 & 0.16 & 0.45 \\
\hline 0.66 & 2.08 & 2.29 & 0.04 & -3.00 & -2.56 \\
\hline-2.45 & -0.72 & 2.18 & -1.43 & -1.85 & 0.41 \\
\hline 0.19 & 2.87 & -1.23 & 2.59 & 2.05 & -1.09 \\
\hline-0.44 & 1.69 & -1.04 & -2.30 & 0.69 & 0.34 \\
\hline 2.55 & -0.76 & 1.41 & -1.80 & 0.79 & -0.54 \\
\hline \multicolumn{6}{|c|}{ Weights $(w)$} \\
\hline FSK2 & FSK4 & FSK8 & FSK16 & FSK32 & FSK64 \\
\hline-2.579 & -7.096 & 0.931 & 0.124 & -0.318 & 8.540 \\
\hline-0.490 & -0.572 & -1.195 & -0.239 & -1.668 & 5.174 \\
\hline-1.532 & -3.564 & -1.195 & 3.627 & 4.663 & -1.570 \\
\hline 6.719 & -5.807 & -1.110 & -0.984 & -2.764 & 0.566 \\
\hline-9.317 & 4.059 & -0.083 & -0.283 & 0.840 & -2.799 \\
\hline-8.051 & -2.456 & 0.068 & 0.990 & 3.381 & -1.628 \\
\hline 0.012 & 3.768 & 0.443 & 0.242 & 4.211 & 12.898 \\
\hline-12.209 & 1.989 & -1.487 & 1.298 & -1.675 & -6.270 \\
\hline 8.351 & 5.954 & -1.451 & -0.296 & -4.969 & 4.876 \\
\hline-4.070 & -0.026 & 0.402 & 1.027 & -5.423 & -8.421 \\
\hline
\end{tabular}

stored. The updated Gabor atom parameters and weights of Gabor filter $\left(c_{i}, \sigma_{i}, f_{i}\right.$, and $\left.w_{i}\right)$ are shown in Tables 1-3. 
TABLE 3: Updated Gabor filter atom parameters and weights for QAM 2-64.

\begin{tabular}{|c|c|c|c|c|c|}
\hline \multicolumn{6}{|c|}{ Shift parameter $(c)$} \\
\hline QAM2 & QAM4 & QAM8 & QAM16 & QAM32 & QAM64 \\
\hline 5.54 & 5.07 & 5.77 & 5.65 & 4.81 & 5.27 \\
\hline 5.77 & 5.90 & 4.25 & 4.45 & 4.18 & 4.29 \\
\hline 4.69 & 4.24 & 4.33 & 5.20 & 4.56 & 5.56 \\
\hline 4.63 & 4.72 & 4.83 & 4.17 & 4.67 & 4.56 \\
\hline 5.02 & 4.29 & 5.00 & 4.08 & 5.13 & 4.59 \\
\hline 5.03 & 5.18 & 5.68 & 5.89 & 5.23 & 4.79 \\
\hline 5.16 & 5.09 & 5.89 & 4.91 & 5.79 & 4.63 \\
\hline 4.61 & 4.67 & 5.52 & 5.91 & 4.35 & 4.47 \\
\hline 4.48 & 4.59 & 5.13 & 5.62 & 5.61 & 4.47 \\
\hline 5.43 & 5.14 & 5.13 & 4.94 & 4.15 & 4.75 \\
\hline \multicolumn{6}{|c|}{ Scale parameter $(\sigma)$} \\
\hline QAM2 & QAM4 & QAM8 & QAM16 & QAM32 & QAM64 \\
\hline 15.63 & 16.25 & 10.25 & 16.76 & 1.67 & 2.45 \\
\hline 16.03 & 8.57 & 8.17 & 5.18 & 13.53 & 16.21 \\
\hline 14.87 & 14.08 & 2.55 & 18.87 & 6.78 & 5.33 \\
\hline 1.92 & 18.27 & 12.31 & 6.63 & 13.33 & 4.66 \\
\hline 6.44 & 3.16 & 11.89 & 18.48 & 9.38 & 5.94 \\
\hline 13.32 & 3.17 & 4.80 & 6.85 & 11.90 & 2.50 \\
\hline 11.49 & 12.94 & 12.83 & 15.33 & 7.44 & 15.29 \\
\hline 14.39 & 3.33 & 7.33 & 7.58 & 6.03 & 5.21 \\
\hline 19.04 & 14.23 & 7.39 & 5.13 & 18.00 & 12.81 \\
\hline 8.67 & 2.94 & 8.48 & 1.05 & 12.56 & 2.59 \\
\hline \multicolumn{6}{|c|}{ Modulation parameter $(f)$} \\
\hline QAM2 & QAM4 & QAM8 & QAM16 & QAM32 & QAM64 \\
\hline 2.35 & -0.05 & 0.80 & 3.14 & -0.53 & 1.82 \\
\hline-2.72 & -0.56 & -0.37 & -0.53 & 2.90 & -3.02 \\
\hline 1.91 & 2.52 & -1.90 & 0.95 & -1.33 & -2.24 \\
\hline-1.02 & 0.10 & -1.55 & 1.46 & 2.47 & 0.38 \\
\hline 1.73 & 1.08 & 1.83 & 1.51 & -0.75 & -1.89 \\
\hline-2.75 & -1.83 & -2.57 & -1.42 & 2.66 & 1.10 \\
\hline 0.47 & 0.20 & -2.79 & -2.21 & 0.54 & -1.57 \\
\hline 0.32 & 0.11 & -0.93 & -1.56 & -0.94 & -0.55 \\
\hline 1.88 & 0.33 & -2.59 & 0.31 & -1.28 & 0.68 \\
\hline-0.76 & -2.27 & 0.07 & 2.83 & -1.01 & -1.70 \\
\hline \multicolumn{6}{|c|}{ Weights $(w)$} \\
\hline QAM2 & QAM4 & QAM8 & QAM16 & QAM32 & QAM64 \\
\hline-0.031 & -0.504 & 1.009 & -1.428 & -1.095 & 0.339 \\
\hline-0.021 & -0.446 & -0.796 & -1.339 & -0.559 & -0.835 \\
\hline-0.043 & -0.187 & -0.285 & -0.725 & 0.304 & 0.442 \\
\hline 0.194 & 0.794 & 0.299 & -1.122 & 1.359 & -0.558 \\
\hline 0.354 & 1.061 & 0.664 & -0.476 & -1.109 & 0.506 \\
\hline 0.250 & -0.591 & -0.306 & 0.044 & -1.357 & -0.234 \\
\hline 0.097 & -0.622 & -0.380 & 1.193 & 0.290 & 0.022 \\
\hline 0.007 & 0.247 & -0.769 & -0.316 & 0.624 & -0.177 \\
\hline 0.245 & 0.467 & -0.608 & 0.984 & -0.334 & 0.694 \\
\hline 0.046 & -0.117 & 0.536 & 0.552 & -0.294 & 0.423 \\
\hline
\end{tabular}

Figures 4-6 show the training of Gabor filter network for different number of iterations in case of PSK modulation, FSK modulation, and QAM, respectively. The training process for different SNRs is also shown in Figures 7-9. The training shows that the mean square error dies down as the number of iterations is increased and also by increasing the SNR. Figures 10-15 show the testing of Gabor filter network for the considered modulations formats (PSK 2 to 64, FSK 2 to 64, and QAM 2 to 64) in the presence of AWGN channel. The probability of correctness is plotted against signal to noise ratio (SNR) and different number of iterations to evaluate the classification accuracy of the proposed Gabor filter network. The simulations results show the classification accuracy for the examples of PSK4, FSK16, and QAM32 which are 100\% for fixed SNR and different number of iterations.

Table 1 shows the updated Gabor atom parameters for the modulation formats of PSK of order 2 to 64 . Table 1 has four parts; the first part shows the updated scale parameter for PSK 2 to 64 , the second part shows the updated shift parameter, the third part shows the updated modulation parameter, and the forth part shows the updated weights of the adaptive filter. All values of updated Gabor filter parameters and weights of adaptive filter are for minimum mean square error.

Table 2 shows the updated Gabor atom parameters for the modulation formats of FSK of order 2 to 64 . Table 2 has four parts; the first part shows the updated scale parameter for FSK 2 to 64 , the second part shows the updated shift parameter, the third part shows the updated modulation parameter, and the forth part shows the updated weights of the adaptive filter. All values of updated Gabor filter parameters and weights of adaptive filter are for minimum mean square error.

Table 3 shows the updated Gabor atom parameters for the modulation formats of QAM of order 2 to 64 . Table 3 has four parts; the first part shows the updated scale parameter for QAM 2 to 64, the second part shows the updated shift parameter, the third part shows the updated modulation parameter, and the forth part shows the updated weights of the adaptive filter. All values of updated Gabor filter parameters and weights of adaptive filter are for minimum mean square error.

Figure 4 shows the training of Gabor filter network for the case of PSK modulation format having order 2 to 64 for different number of iterations at fixed SNR of $10 \mathrm{~dB}$. The parameters of Gabor filter network are trained for greater than 50 iterations for the case of PSK 2, 4, and 8, while for the PSK 16, 32, and 64 the training of Gabor filter network is for less than 50 iterations. The mean square error is minimized and approaches to zero for all curves shown in Figure 4, as a number of iterations are increased.

Figure 5 shows the Gabor filter network training for the case of FSK modulation format having order 2 to 64 for fixed number of iterations. As shown in Figure 5, the mean square error is approaching to zero, when a number of iterations are increased. The training of Gabor filter network is done for maximum 50 iterations for the FSK modulation case.

Figure 6 shows the training of Gabor filter network for QAM case with fixed SNR of $10 \mathrm{~dB}$ and different iterations. The training of Gabor filter network shows minimized mean square error for all curves shown in Figure 6 with less number of iterations. In Figure 6, QAM 16, 32, and 64 are trained for 


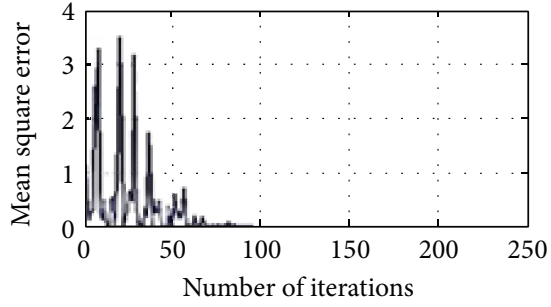

— PSK2

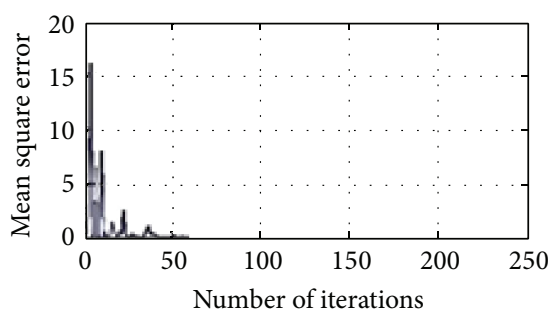

— PSK16

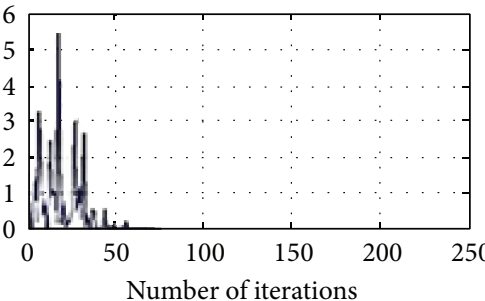

— PSK4

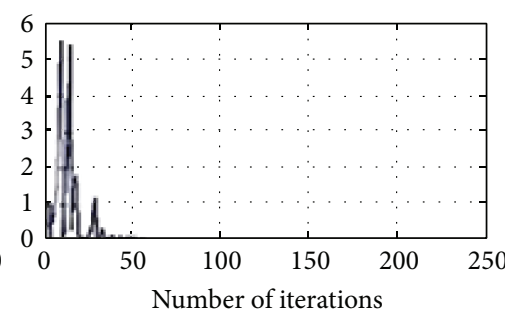

— PSK32

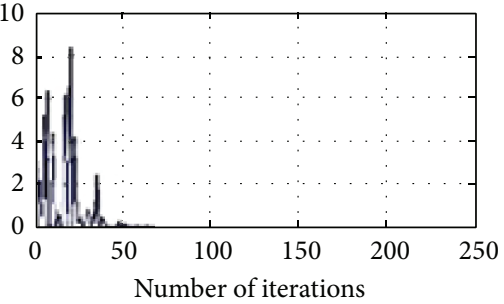

— PSK8

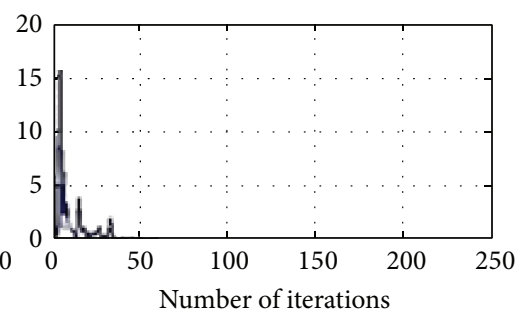

— PSK64

FIGURE 4: Training of Gabor filter parameters and weights for modulation classification in case of PSK modulation 2-64 for different number of iterations at $\mathrm{SNR}=10 \mathrm{~dB}$.

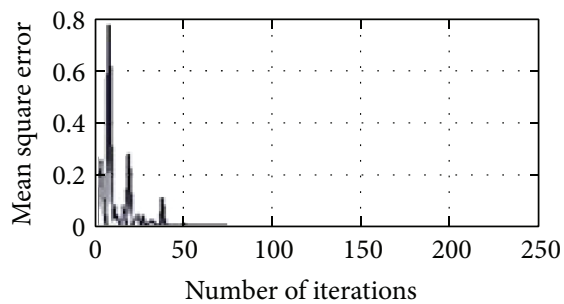

— FSK2

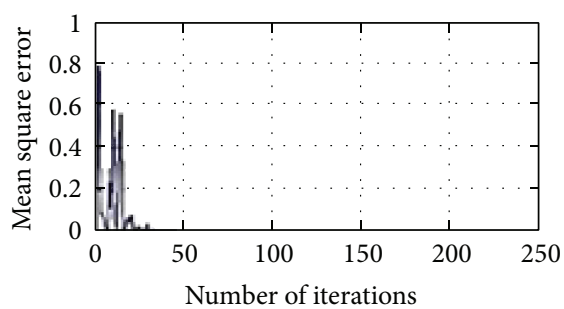

— FSK16
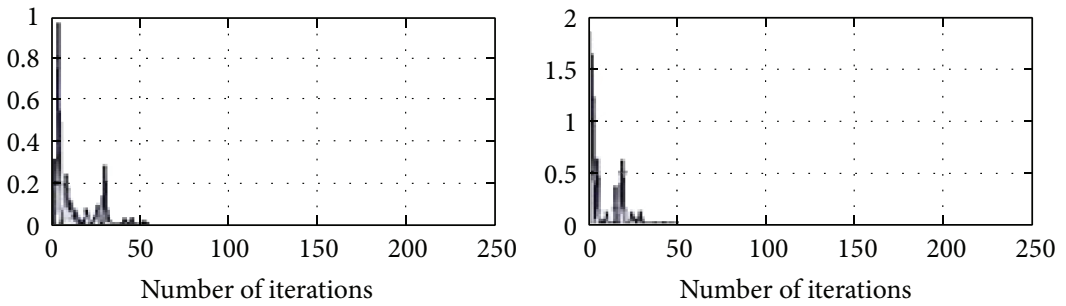

- FSK4

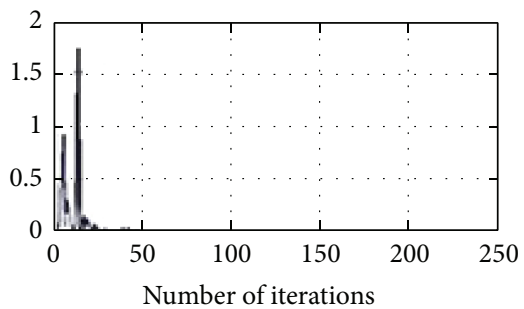

— FSK32

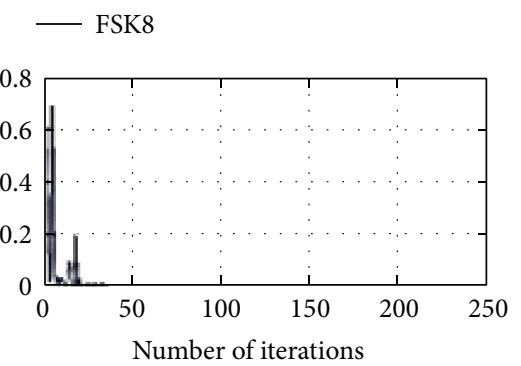

- FSK64

FIGURE 5: Training of Gabor filter parameters and weights for modulation classification in case of FSK modulation 2-64 for different number of iterations at $\mathrm{SNR}=10 \mathrm{~dB}$.

20 iterations and QAM 2, 4, and 8 are trained for above 50 iterations.

Figure 7 shows the training of Gabor filter network parameters and weights of adaptive filter in case of PSK modulation up to order 2 to 64 with fixed number of iterations and different SNRs. As signal to noise ratio is varied from 0 to 20 , the mean square error approaching towards zero. The training of proposed algorithm for all cases of considered modulation is done successfully and Figure 7 shows that the proposed algorithm for the modulation classification is trained at SNR of $10 \mathrm{~dB}$.

Figure 8 shows the training of Gabor filter network parameters and weights of adaptive filter in case of FSK modulation up to order 2 to 64 with fixed number of iterations and different SNRs. The training of FSK modulation formats is done at SNR of $10-15 \mathrm{~dB}$.

The training of Gabor filter network for QAM 2 to 64 at different SNRs and fixed number of iterations is shown in 


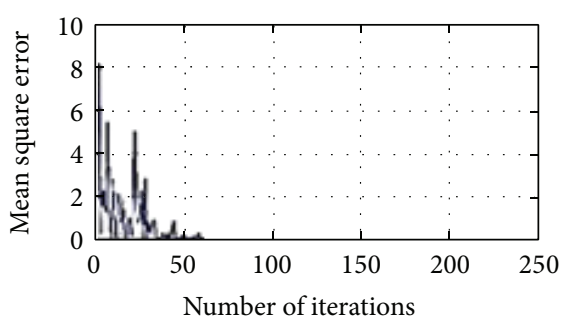

- QAM2

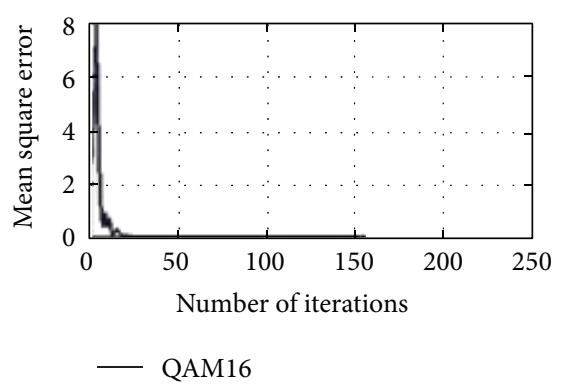

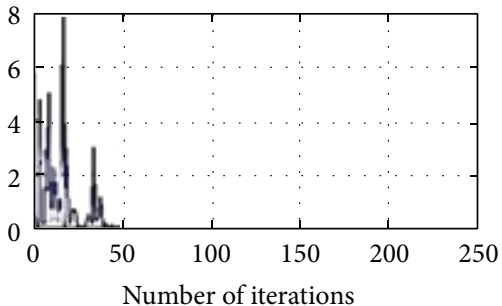

- QAM4

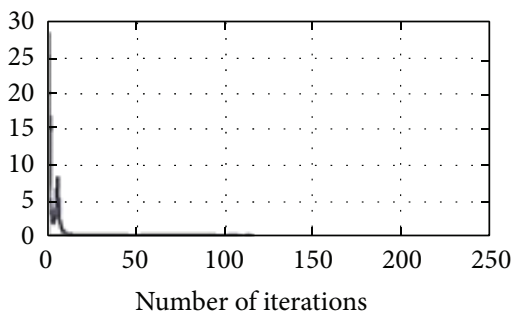

- QAM32

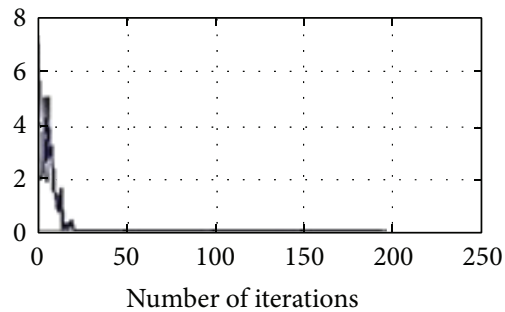

- QAM8

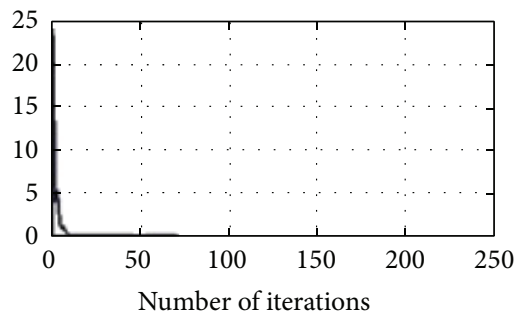

- QAM64

FIGURE 6: Training of Gabor filter parameters and weights for modulation classification in case of QAM 2-64 for different number of iterations at $\mathrm{SNR}=10 \mathrm{~dB}$.

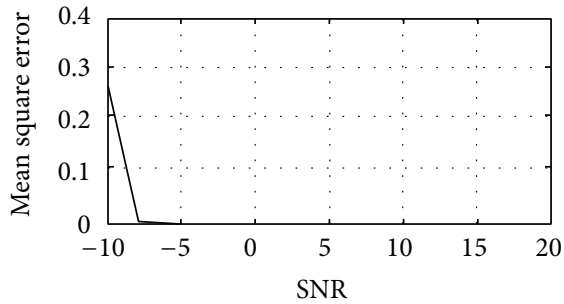

- FSK2

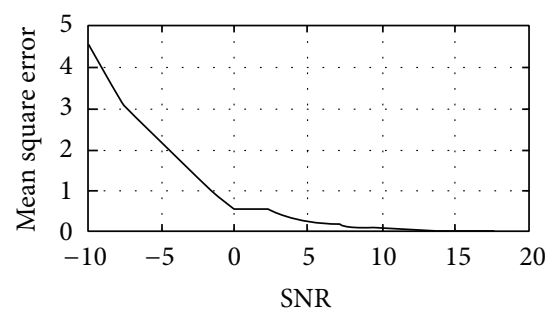

— FSK16

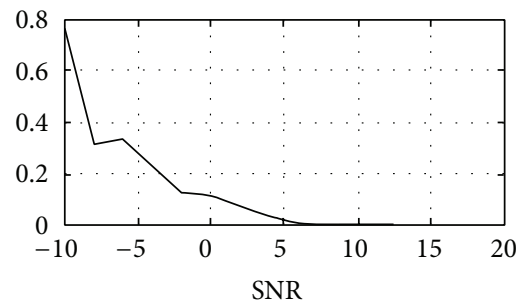

- FSK4

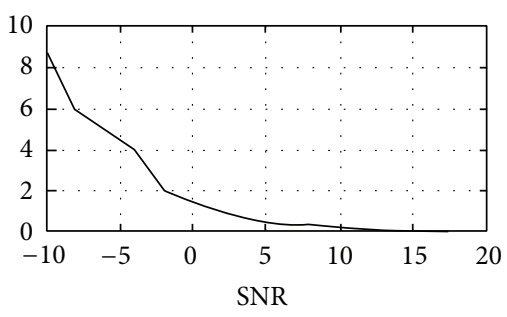

- FSK32

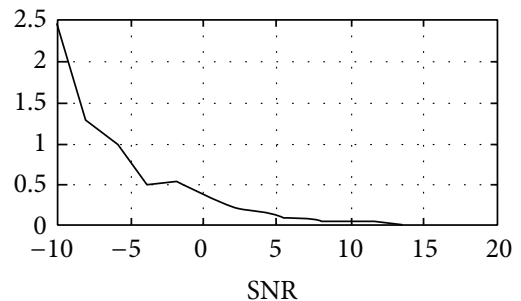

FSK8

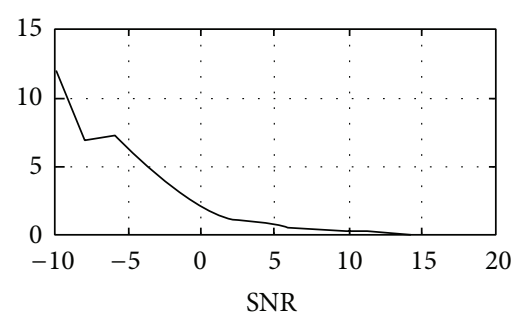

- FSK64

FIGURE 7: Training of Gabor filter parameters and weights for modulation classification in case of PSK modulation 2-64 at different SNRs and fixed number of iterations.

Figure 9. The parameters of Gabor filter network and weights of the adaptive filter are updated and mean square error is minimized as SNR is increased from 0 to $20 \mathrm{~dB}$.

The example considered in Figures 10 and 11 is PSK4. The probability of correctness versus different number of iterations at SNR $=10 \mathrm{~dB}$ is shown in Figure 10, while PCC curve versus SNR at fixed number of iterations is shown in Figure 11. The PCC in Figure 10 is approximately 1 when the number of iterations is increased up to 200. From Figures 10 and 11, the classification performance of Gabor filter network for the PSK modulation scenario under the effect of white
Gaussian noise channel is approximately $100 \%$ at lower SNR. In Figures 10 and 11, the example considered are PSK 4 and it is shown form the results that PSK4 classified correctly among class of PSK modulation formats having order 2 to 64 .

In Figures 12 and 13, the probability of correctness for FSK modulation scenario is shown for different number of iterations and SNR. The example considered is FSK16. The PCC curve shows that the classification performance is approximately $100 \%$ at $\mathrm{SNR}=15 \mathrm{~dB}$ for fixed number of iterations. The probability of correctness is approximately 1 in Figure 12, when a number of iterations are increased up to 

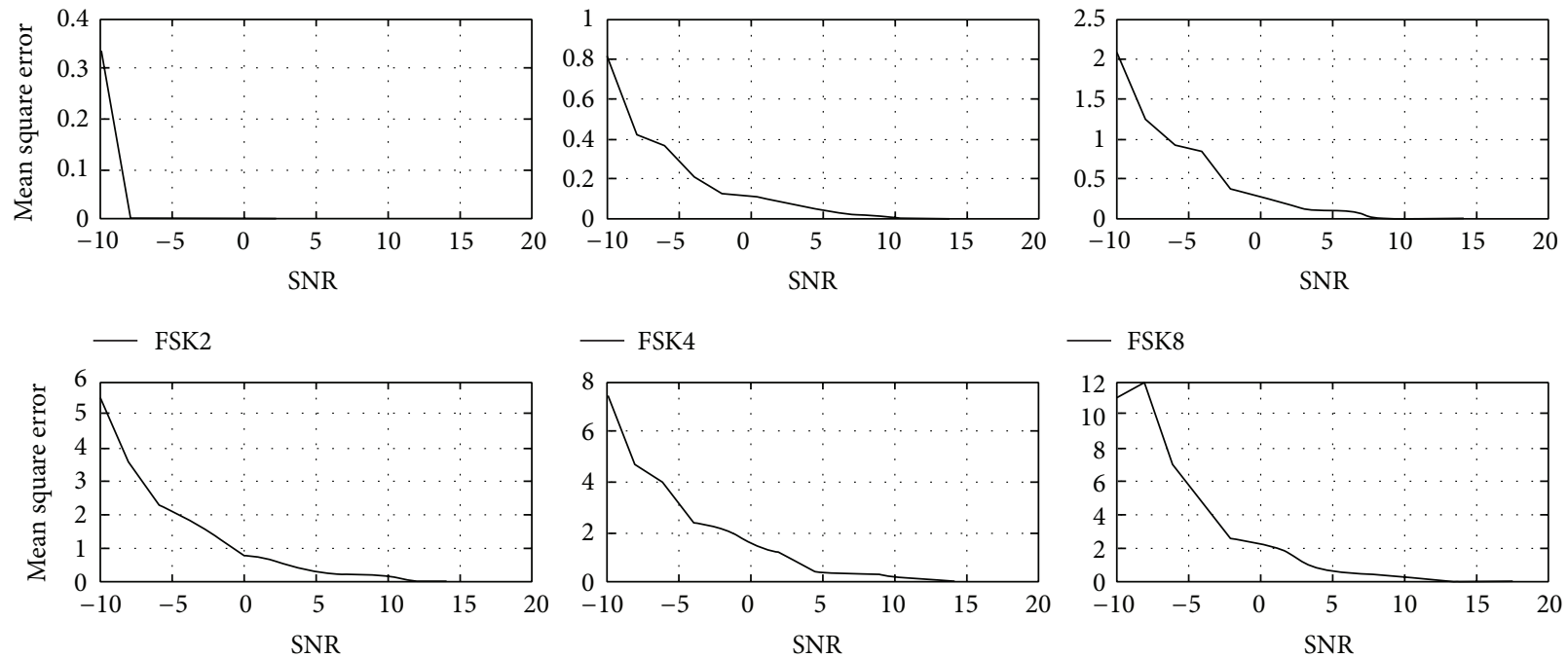

- FSK16

- FSK32

— FSK64

FIGURE 8: Training of Gabor filter parameters and weights for modulation classification in case of FSK modulation 2-64 at different SNRs and fixed number of iterations.
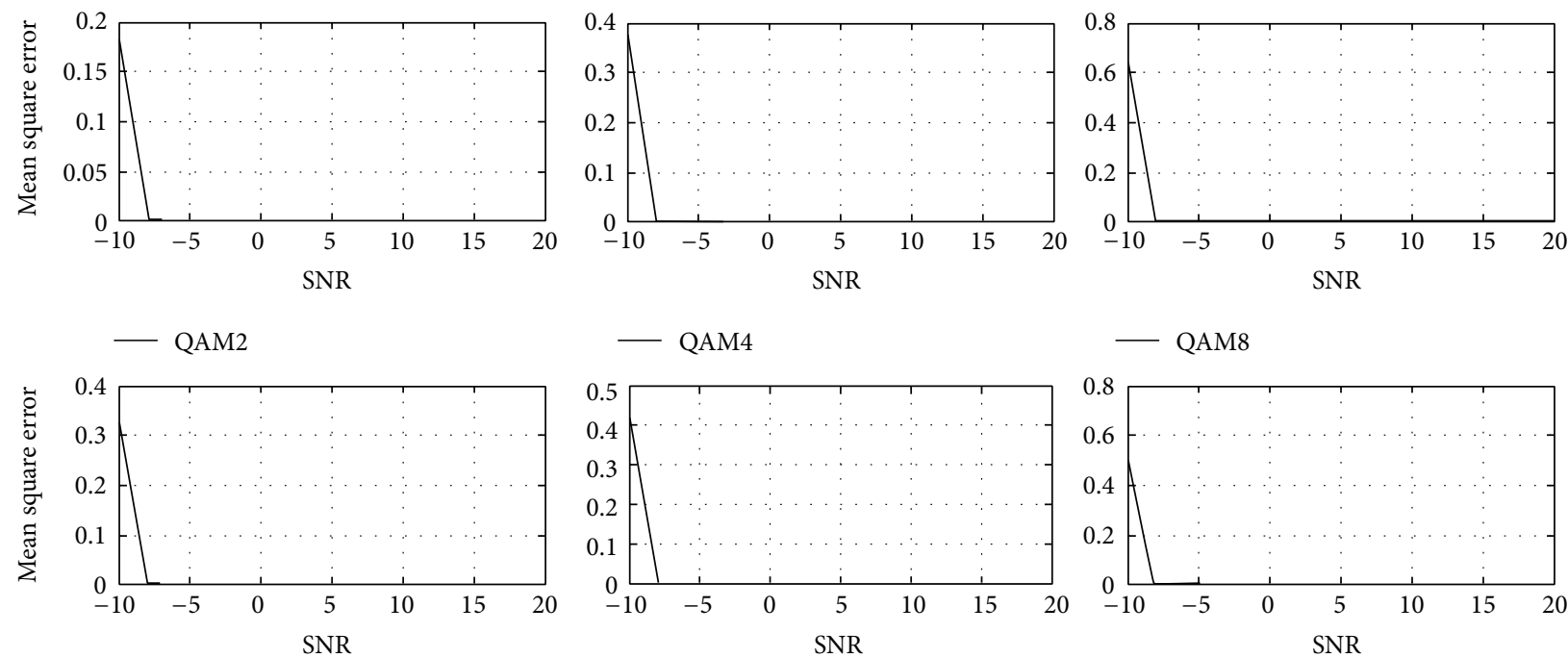

— QAM16

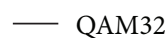

- QAM64

FIGURE 9: Training of Gabor filter parameters and weights for modulation classification in case of QAM 2-64 at different SNRs and fixed number of iterations.

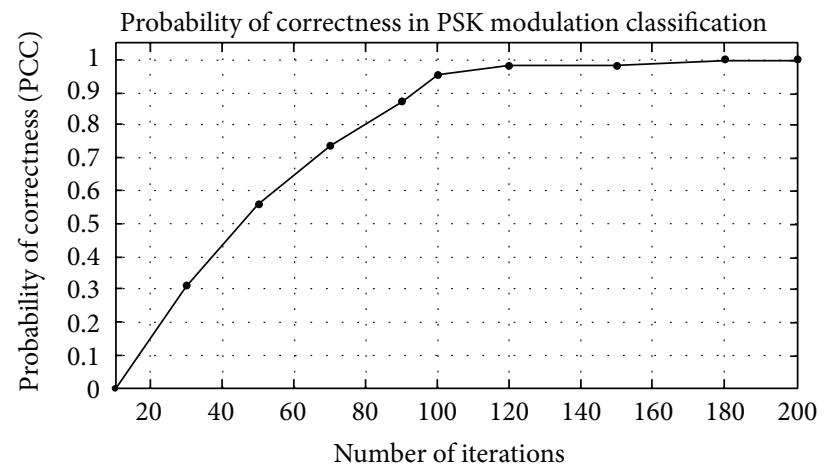

FIGURE 10: Probability of correctness (PCC) versus number of iterations at SNR $=10 \mathrm{~dB}$ for PSK4 example. 


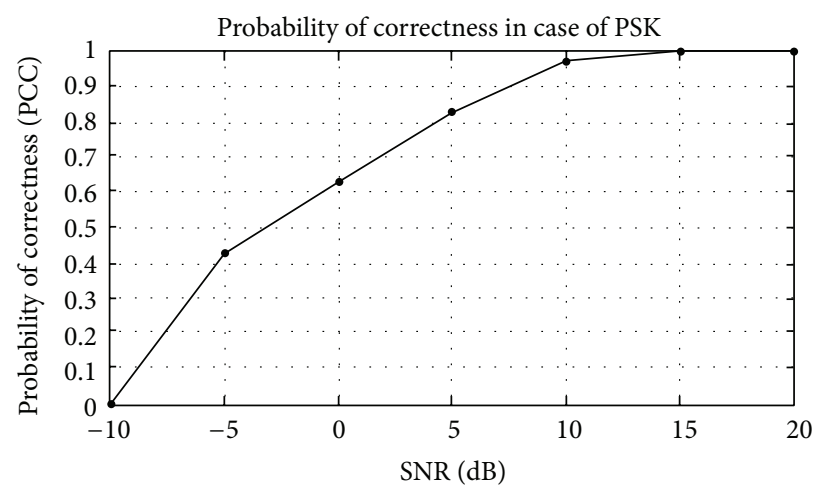

Figure 11: Probability of correctness (PCC) versus SNR for fixed number of iterations in PSK4 example.

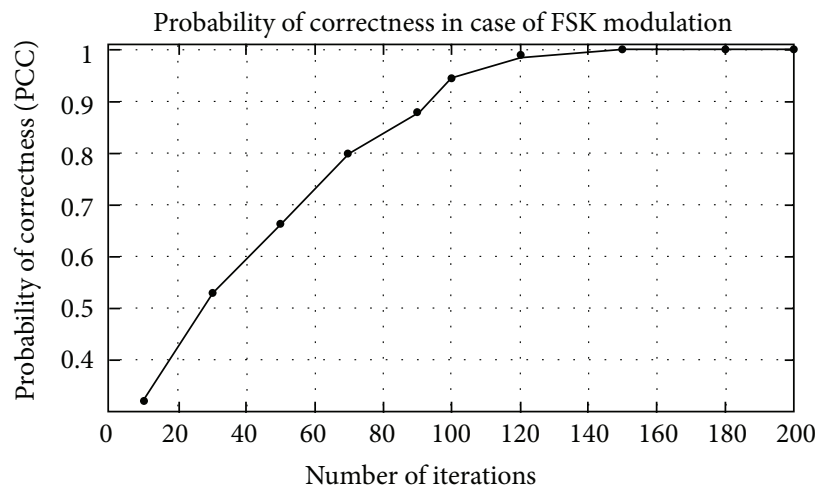

FIGURE 12: Probability of correctness (PCC) versus number of iterations at SNR $=10 \mathrm{~dB}$ for FSK16 example.

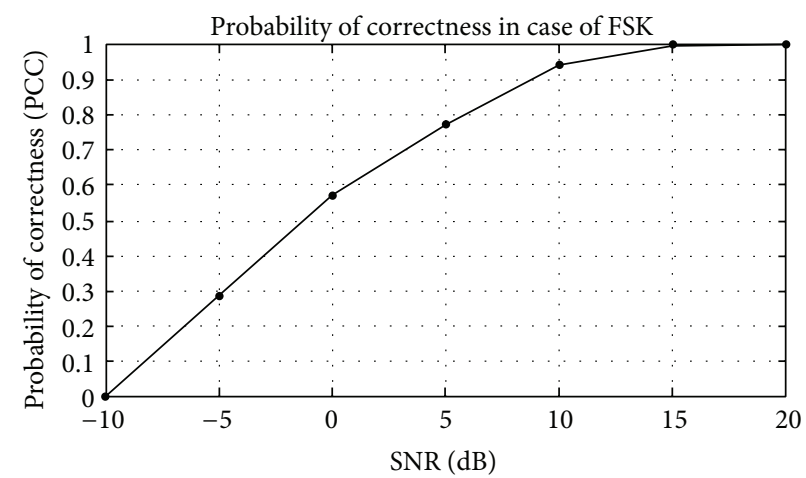

FIGURE 13: Probability of correctness (PCC) versus SNR for fixed number of iterations in FSK16 example.

200. The FSK 16 example is classified accurately among the group of considered modulation formats.

In Figures 14 and 15, the example considered is QAM32 for classification purpose. The classification curve in the form of PCC versus different number of iterations at fixed SNR of $10 \mathrm{~dB}$ is shown in Figure 14, while in Figure 15 PCC curves versus SNR at fixed number of iterations are shown. The classification of Gabor filter network in case of QAM is quite better with the existing modulation classification techniques.

The simulation results show the $100 \%$ classification accuracy of the proposed algorithm. The features extracted from the proposed architecture and classifier based upon Gabor filter network provide correct classification among group of considered modulation formats. Moreover, the received signal is corrupted by additive white Gaussian noise but the classification accuracy is approximately $100 \%$ at lower SNRs. The algorithm is also computationally less complex and classification accuracy is attained at less number of iterations.

\section{Conclusion}

In this paper, proposed classifier which is Gabor filter based is used for feature extraction and also for the classification purpose. The Gabor filter input layer constitutes the feature extraction part $\left(c_{i}, \sigma_{i}\right.$, and $\left.f_{i}\right)$, whereas weights $\left(w_{i}\right)$ between 


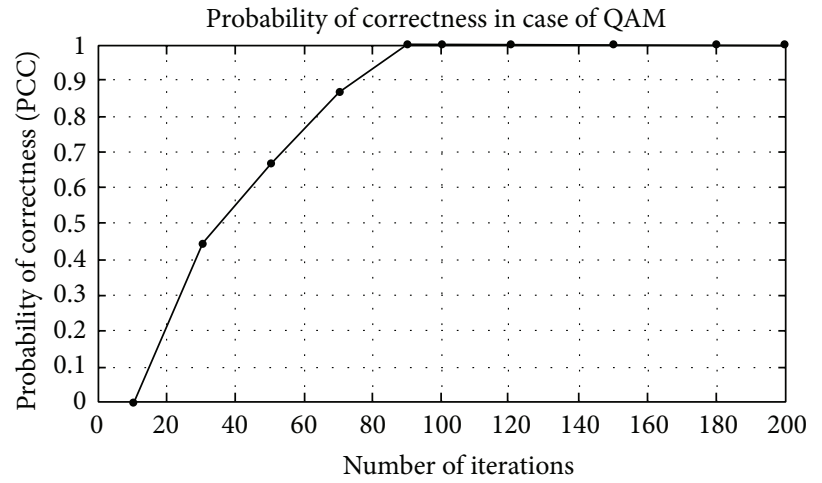

FIGURE 14: Probability of correctness (PCC) versus number of iterations at SNR $=10 \mathrm{~dB}$ for QAM32 example.

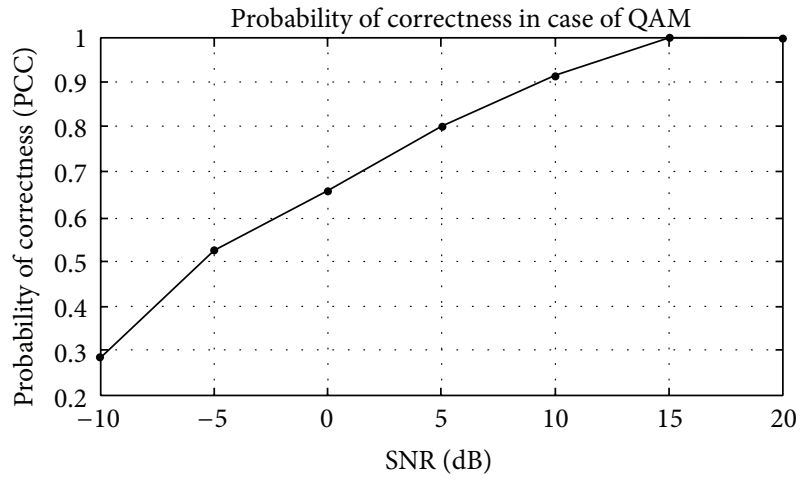

FIGURE 15: Probability of correctness (PCC) versus SNR for fixed number of iterations in QAM32 example.

Gabor atom nodes and output of the Gabor filter constitute the linear classification part. The feature extraction layer and classification part search for the optimal Gabor atom parameters and weights of the Gabor filter, so that error is to be minimized. The considered modulations such as PSK2, PSK4, PSK8, PSK16, PSK32, PSK64, QAM2, QAM4, QAM8, QAM 16, QAM 32, QAM 64, FSK2, FSK4, FSK8, FSK16, FSK32, and FSK64 are classified under the effects of AWGN channel. The classifier proposed here is very effective performance in considered scenarios of modulation. The proposed novel Gabor filter based modulation classification technique shows $100 \%$ classification accuracy at lower SNR.

\section{Conflict of Interests}

The authors declare that there is no conflict of interests regarding the publication of this paper.

\section{References}

[1] S. A. Ghauri, I. M. Qureshi, A. N. Malik, and T. A. Cheema, "Higher order cummulants based digital modulation recognition scheme," Research Journal of Applied Sciences Engineering \& Technology, vol. 6, no. 20, pp. 3910-3915, 2013.

[2] S. A. Ghauri and I. M. Qureshi, "Automatic classification of digital modulated signals using linear discriminant analysis on AWGN channel," in Proceedings of the 1st International Conference on Information and Communication Technology Trends (ICICTT '13), September 2013.

[3] T. Yücek and H. Arslan, "A novel sub-optimum maximumlikelihood modulation classification algorithm for adaptive OFDM systems," in Proceedings of the IEEE Wireless Communications and Networking Conference (WCNC '04), vol. 2, pp. 739-744, March 2004.

[4] P. Panagiotou, A. Anastasopoulos, and A. Polydoros, "Likelihood ratio tests for modulation classification," in Proceedings of the 21st Century Military Communications (MILCOM '00), vol. 2, pp. 670-674, Los Angeles, Calif, USA, October 2000.

[5] W. Wei and J. M. Mendel, "Maximum-likelihood classification for digital amplitude-phase modulations," IEEE Transactions on Communications, vol. 48, no. 2, pp. 189-193, 2000.

[6] P. Marchand, C. Le Martret, and J. Lacoume, "Classification of linear modulation by a combination of different orders cyclic cumulants," in Proceedings of the IEEE Signal Processing Workshop on Higher-Order Statistics (SPW-HOS '97), pp. 47-51, July 1997.

[7] O. A. Dobre, Y. Bar-Ness, and W. Su, "Higher-order cyclic cummulants for high order modulation classification," in Proceedings of the IEEE Military Communications Conference (MILCOM '03), vol. 1, pp. 112-117, Boston, Mass, USA, October 2003.

[8] E. E. Azzouz and A. K. Nandi, "Procedure for automatic recognition of analogue and digital modulation," IEE ProceedingsCommunications, vol. 143, no. 5, pp. 259-266, 1996.

[9] B. Ramkumar, "Automatic modulation classification for cognitive radios using cyclic feature detection," IEEE Circuits and Systems Magazine, vol. 9, no. 2, pp. 27-45, 2009.

[10] S. Xi and H. C. Wu, "Robust automatic modulation classification using cumulant features in the presence of fading channels," in Proceedings of the IEEE Wireless Communications and Networking Conference (WCNC '06), vol. 4, pp. 2094-2099, April 2006.

[11] H. Wu, M. Saquib, and Z. Yun, "Novel automatic modulation classification using cumulant features for communications via multipath channels," IEEE Transactions on Wireless Communications, vol. 7, no. 8, pp. 3098-3105, 2008.

[12] L. Qian and C. Zhu, "Modulation classification based on cyclic spectral features and neural network," in Proceedings of the $3 r d$ International Congress on Image and Signal Processing (CISP '10), pp. 3601-3605, Yantai, China, October 2010.

[13] M. L. D. Wong and A. K. Nandi, "Automatic digital modulation recognition using artificial neural network and genetic algorithm," Signal Processing, vol. 84, no. 2, pp. 351-365, 2004. 
[14] V. D. Orlic and M. L. Dukic, "Automatic modulation classification algorithm using higher-order cumulants under real-world channel conditions," IEEE Communications Letters, vol. 13, no. 12, pp. 917-919, 2009.

[15] P. Prakasam and M. Madheswaran, "Digital modulation identification model using wavelet transform and statistical parameters," Journal of Computer Systems, Networks, and Communications, vol. 2008, Article ID 175236, 8 pages, 2008.

[16] Z. Wu, E. Like, V. D. Chakravarthy, and P. Ratazzi, "Signal classification in fading channels using cyclic spectral analysis," Eurasip Journal on Wireless Communications and Networking, vol. 2009, Article ID 879812, pp. 1-14, 2009.

[17] W. Hamouda, K. Hassan, I. Dayoub, and M. Berbineau, "Automatic modulation recognition using wavelet transform and neural networks in wireless systems," Eurasip Journal on Advances in Signal Processing, vol. 2010, Article ID 532898, 2010.

[18] O. A. Dobre, S. Rajan, and R. Inkol, "Joint signal detection and classification based on first-order cyclostationarity for cognitive radios," Eurasip Journal on Advances in Signal Processing, vol. 2009, Article ID 656719, 2009.

[19] V. Le Nir, T. Van Waterschoot, J. Duplicy, and M. Moonen, "Blind coarse timing offset estimation for CP-OFDM and ZPOFDM transmission over frequency selective channels," Eurasip Journal on Wireless Communications and Networking, vol. 2009, Article ID 262813, 2009.

[20] M. Gandetto, M. Guainazzo, and C. S. Regazzoni, "Use of time frequency analysis and neural networks for mode identification in a wireless software defined radio approach," EURASIP Journal on Applied Signal Processing, pp. 1778-1790, 2014.

[21] Y. Zhang, N. Ansari, and W. Su, "Multi-sensor signal fusion based modulation classification by using wireless sensor networks," in Proceedings of the IEEE International Conference on Communications (ICC '11), pp. 1-5, Kyoto, Japan, June 2011.

[22] X. Zhou, Y. Wu, and B. Yang, "Signal classification method based on SVM and higher order cummulatnts," Wireless Sensor Networks, vol. 2, pp. 48-52, 2010.

[23] L. Cheng and J. Liu, "An optimal neural network classifier for automatic modulation recognition," TELKOMNIKA Indonesian Journal of Electrical Engineering, vol. 12, no. 2, pp. 1343-1352, 2014.

[24] M. Luo, L. Li, G. Qian, and J. Lu, "A blind modulation identification algorithm for STBC systems using multidimensional ICA," Concurrency Computation Practice and Experience, vol. 26, no. 8, pp. 1490-1505, 2014.

[25] J. Liu, Z. Dong, K. P. Zhong et al., "Modulation format identification based on received signal power distributions for digital coherent recivers," in Proceedings of the Optical Fiber Conferences, pp. 9-13, 2014.

[26] J. Sanderson, X. Li, Z. Q. Liu, and Z. Q. Wu, "Hierarchical blind modulation classification for underwater acoustic communication signal via cyclostationary and maximal likelihood analysis," in Proceedings of the IEEE Military Communications Conference (MILCOM '13), pp. 29-34, 2013.

[27] E. Soltan Mohammadi and M. Naraghi-Por, "Blind modulation classification over fading channels using expectation maximization," IEEE Communication Letters, vol. 17, no. 9, pp. 1692-1695, 2013.

[28] Y. Liu, O. Simeone, A. M. Haimouich, and W. Su, "Modulation classification via Gibbs sampling based on a Latent Dirichlet Bayesian Network," IEEE Signal Processing Letters, vol. 21, no. 9, pp. 1135-1139, 2014.
[29] M. Marcy and O. A. Dobre, "Blind modulation classification for single and multiple antenna system over frequency selective channels," IEEE Signal Processing Letters, vol. 21, no. 9, pp. 10981102, 2014.

[30] J. P. Jide, "Automatic recognition of both inter and intra classes of digital modulated signals using artificial neural netowrk," Journal of Engineering Science and Technology, vol. 9, no. 2, pp. 273-285, 2014.

[31] B. Dulek, O. Ozdemir, P. K. Varshney, and W. Su, "A novel approach to dictionary construction for automatic modulation classification," Journal of the Franklin Institute, vol. 351, no. 5, pp. 2991-3012, 2014

[32] J. Zhang, G. G. Walter, Y. Miao, and W. N. W. Lee, "Wavelet neural networks for function learning," IEEE Transactions on Signal Processing, vol. 43, no. 6, pp. 1485-1497, 1995.

[33] S. Qain and D. Chen, "Joint time frequency analysis," Signal Processing Magazine, vol. 16, pp. 52-67, 1999.

[34] T. G. Sayan, K. Leblebicioglu, and T. Ince, "Electromagnetic target classification using time-frequency analysis and neural networks," Microwave and Optical Technology Letters, vol. 21, no. 1, pp. 63-69, 1999.

[35] Y. Shi and X. Zhang, "A Gabor atom network for signal classification with application in radar target recognition," IEEE Transactions on Signal Processing, vol. 49, no. 12, pp. 2994-3004, 2001.

[36] F. Zhu, X. Zhang, and Y. Hu, "Gabor filter approach to joint feature extraction and target recognition," IEEE Transactions on Aerospace and Electronic Systems, vol. 45, no. 1, pp. 17-30, 2009. 

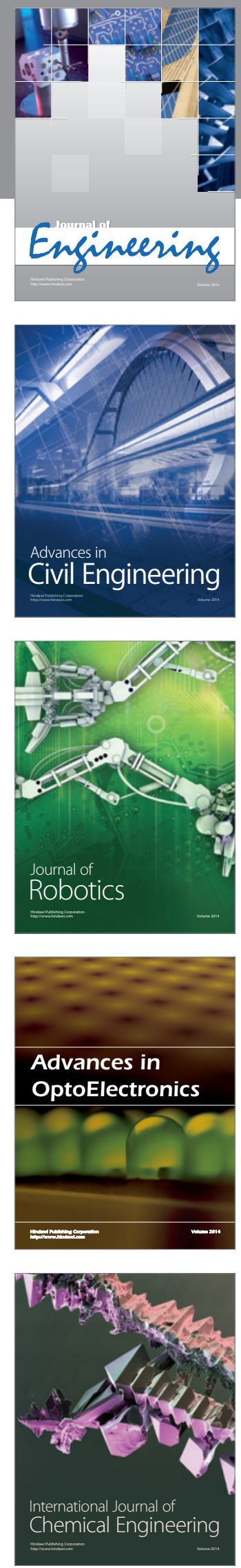

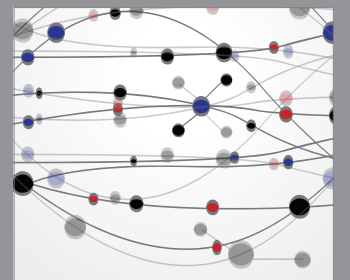

The Scientific World Journal
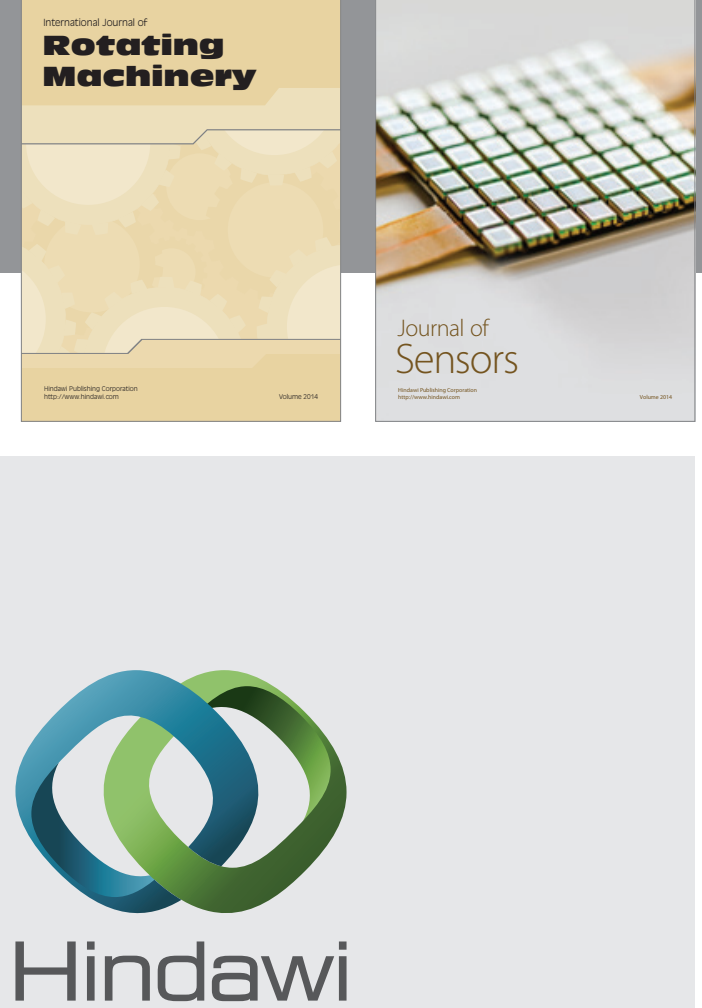

Submit your manuscripts at http://www.hindawi.com
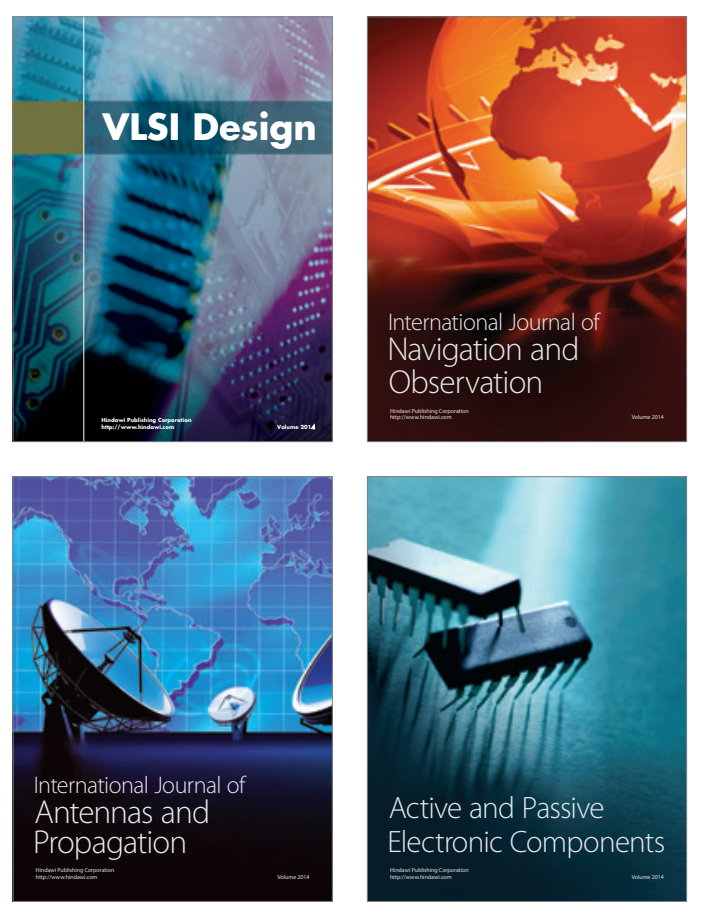
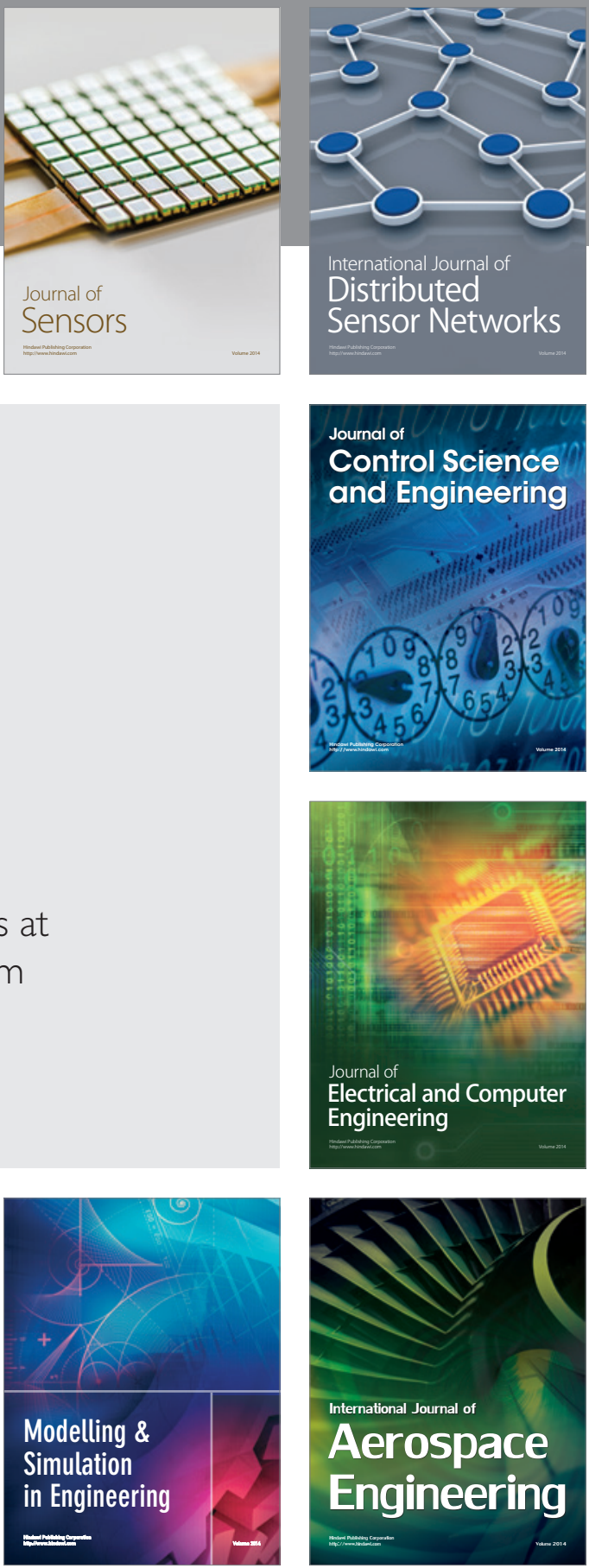

Journal of

Control Science

and Engineering
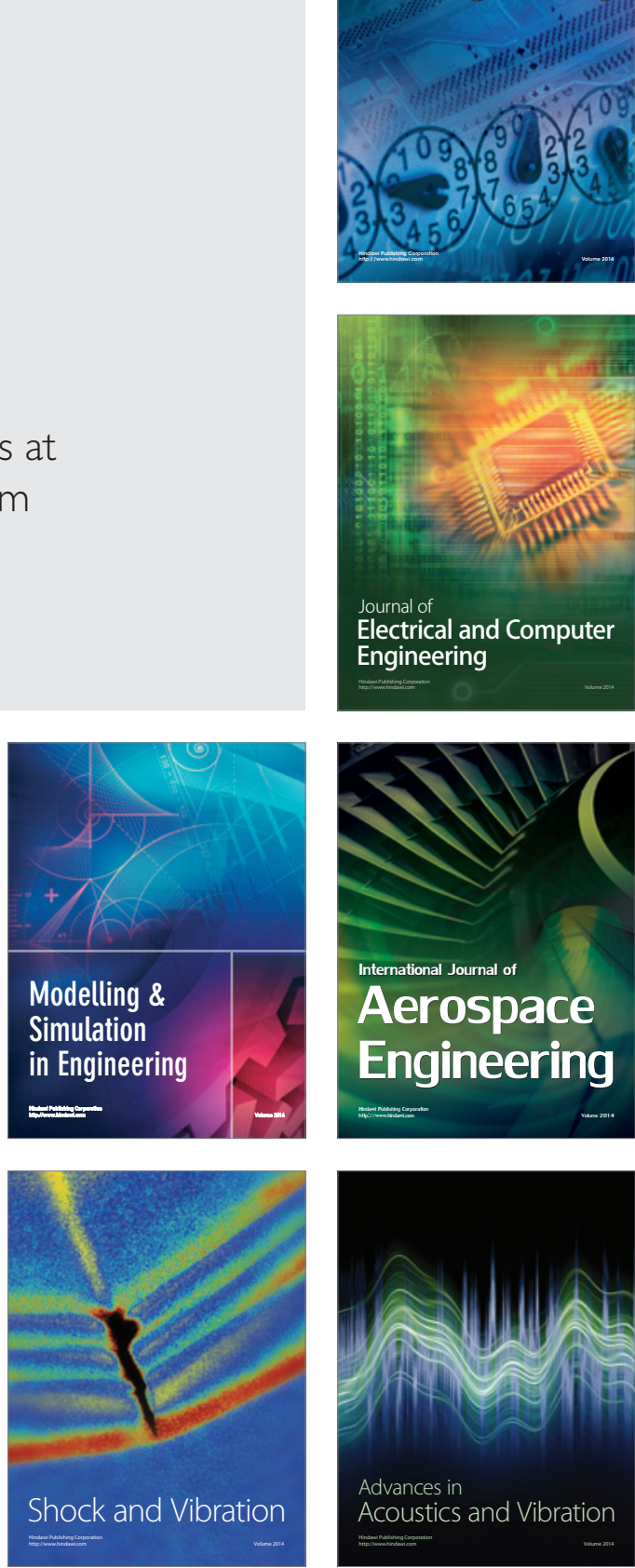